\title{
A method to track rotational motion for use in single-molecule biophysics
}

\author{
Jan Lipfert, ${ }^{\text {a) }}$ Jacob J. W. Kerssemakers, ${ }^{\text {a) }}$ Maylon Rojer, and Nynke H. Dekker ${ }^{\text {b) }}$ \\ Department of Bionanoscience, Kavli Institute of Nanoscience, Delft University of Technology, \\ Lorentzweg 1, 2628 CJ Delft, The Netherlands
}

(Received 21 July 2011; accepted 21 September 2011; published online 25 October 2011)

\begin{abstract}
The double helical nature of DNA links many cellular processes such as DNA replication, transcription, and repair to rotational motion and the accumulation of torsional strain. Magnetic tweezers (MTs) are a single-molecule technique that enables the application of precisely calibrated stretching forces to nucleic acid tethers and to control their rotational motion. However, conventional magnetic tweezers do not directly monitor rotation or measure torque. Here, we describe a method to directly measure rotational motion of particles in MT. The method relies on attaching small, non-magnetic beads to the magnetic beads to act as fiducial markers for rotational tracking. CCD images of the beads are analyzed with a tracking algorithm specifically designed to minimize crosstalk between translational and rotational motion: first, the in-plane center position of the magnetic bead is determined with a kernel-based tracker, while subsequently the height and rotation angle of the bead are determined via correlation-based algorithms. Evaluation of the tracking algorithm using both simulated images and recorded images of surface-immobilized beads demonstrates a rotational resolution of $0.1^{\circ}$, while maintaining a translational resolution of 1-2 $\mathrm{nm}$. Example traces of the rotational fluctuations exhibited by DNA-tethered beads confined in magnetic potentials of varying stiffness demonstrate the robustness of the method and the potential for simultaneous tracking of multiple beads. Our rotation tracking algorithm enables the extension of MTs to magnetic torque tweezers (MTT) to directly measure the torque in single molecules. In addition, we envision uses of the algorithm in a range of biophysical measurements, including further extensions of MT, tethered particle motion, and optical trapping measurements. @ 2011 American Institute of Physics. [doi:10.1063/1.3650461]
\end{abstract}

\section{INTRODUCTION}

Magnetic tweezers (MTs) are a powerful single-molecule technique to study the physical properties, dynamics, and interactions of biological macromolecules. ${ }^{1-4}$ MT assays have provided unique insights into the function and dynamics of biological macromolecules. Examples include studies of the properties of bare DNA, ${ }^{5,6} \mathrm{RNA}^{7}$ and of enzymes that act on DNA or RNA, such as topoisomerases, ${ }^{8}$ helicases,,${ }^{4,9}$ and polymerases. ${ }^{10}$

In a typical configuration (Fig. 1(a)), a DNA or RNA molecule is attached at one end to the surface of a flow cell and at the other end to a superparamagnetic bead that is manipulated by external magnetic fields. ${ }^{2,11,12}$ Conventional MT implementations typically track the $\mathrm{x}, \mathrm{y}$, and $\mathrm{z}$ positions of tethered superparamagnetic particles from analysis of CCD images. The applied stretching force can be calibrated by analyzing the fluctuations in the $\mathrm{x}$ and $\mathrm{y}$ positions. ${ }^{5,12,13}$ In addition, magnetic tweezers allow rotation of the tethered particles by rotating the magnetic fields. A sufficient amount of twist will trigger torsional buckling and the subsequent formation of plectonemic supercoils. ${ }^{5,11}$

Recently, several approaches have been developed to track the rotation angle in MT measurements in addition to the bead's position. Rotational tracking is useful for the study of rotary motion in biological processes, e.g., molecular motion

a) J. Lipfert and J. J. W. Kerssemakers contributed equally to this work.

b) Author to whom correspondence should be addressed. Electronic mail: N.H.Dekker@tudelft.nl. that results in DNA over- or underwinding. In addition, analysis of the angular fluctuations allows for the measurement of torque. ${ }^{14-16}$ Several rotational tracking methods rely on fluorescence excitation and detection; ${ }^{17,18}$ some require the use of internal modification of the DNA tether ${ }^{18}$ or specially nanofabricated particles. ${ }^{14}$

Here we describe in detail a method to track the $\mathrm{x}, \mathrm{y}$, and $\mathrm{z}$ positions and the rotation angle that is designed for its simplicity, staying close to the approach that has become standard in MT experiments. Our strategy is based on attaching small, non-magnetic beads that act as fiducial markers to larger magnetic beads. For the (x,y)-position tracking of these magnetic beads, we minimize the influence of the fiducial marker by using a kernel-based tracker that achieves spatial filtering on circularly symmetric patterns. Next, the angular coordinate is determined by transforming the marked-bead images from Cartesian (x, y) to polar coordinates (r, $\theta$ ) and using a correlation-based tracking routine to determine the rotation angle. The z-position, i.e., the beads' height, is tracked employing a cross-correlation tracker based on look-up tables recorded at different focal planes. ${ }^{19}$ We note that the algorithm that we introduce for angular tracking is fully compatible with simultaneous $\mathrm{x}, \mathrm{y}$, and $\mathrm{z}$ tracking: analysis of outof-focus images of the marked beads using the kernel-based tracker permits $\mathrm{x}, \mathrm{y}$, and $\mathrm{z}$ tracking with an accuracy comparable to conventional MT measurements employing unmarked beads.

We have recently demonstrated the use of such a tracking algorithm to enable straightforward torque measurements on DNA and nucleo-protein filaments ${ }^{15}$ in magnetic torque 


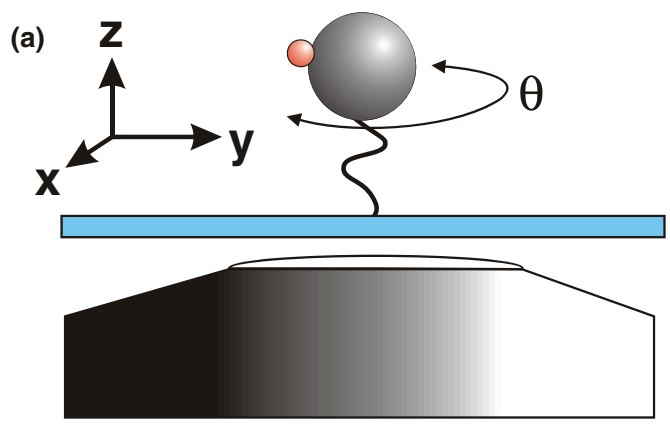

(b)

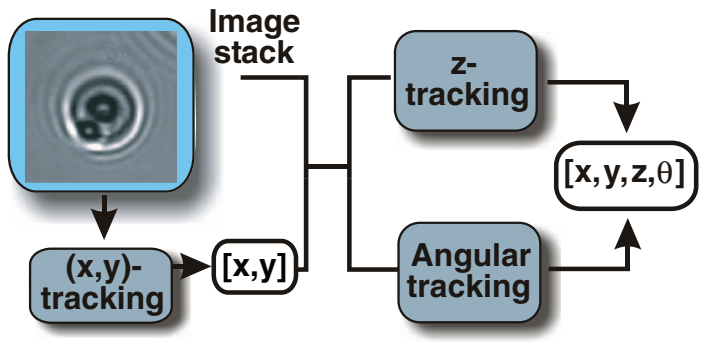

(c)

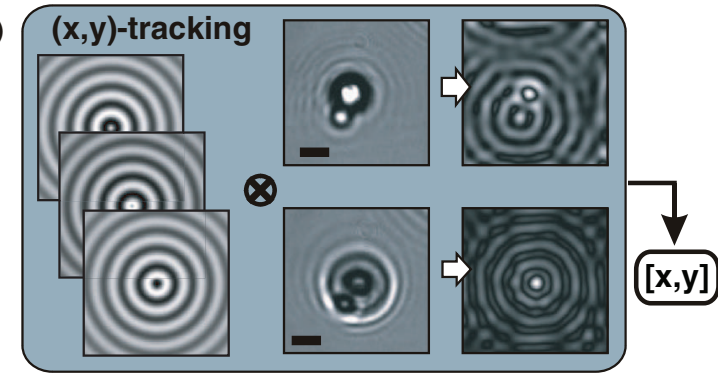

FIG. 1. (Color online) Schematic of magnetic tweezers rotation measurements and overview of the tracking algorithm. (a) Experimental setup for imaging transverse, vertical, and rotational motion of a tethered magnetic bead with a smaller fiducial marker bead attached. (b) Flowchart of position $(\mathrm{x}, \mathrm{y}, \mathrm{z})$ and rotation $(\theta)$ tracking of bead motion via microscope images. (c) Use of circular kernel patterns for transverse (x, y)-position tracking and spatial filtering. Cross-correlation of bead images with the kernel patterns creates correlation maps with separated maxima for the main bead and the fiducial bead. Using out of focus images (lower panels) causes the main bead maximum to dominate the correlation pattern, thus effectively eliminating disturbances in the determination of the main bead's symmetry center by the fiducial bead.

tweezers (MTT). Given the presence of an appropriately chosen magnetic field configuration, such MTT maintain the advantages of conventional MT, namely, a large force range, absence of radiation damage, the ability to precisely calibrate stretching forces, and facile implementation, while in addition permitting measurements of the torque stored in biological molecules. In addition to its use in magnetic tweezers measurements, we expect that this tracking algorithm can find application in tethered particle motion measurements ${ }^{20}$ and in optical tweezers measurements, especially as the latter rely increasingly on camera-based tracking. ${ }^{21}$

\section{METHODS AND MATERIALS}

\section{A. Magnetic tweezers experimental configuration}

Our MT setup employed a custom-built inverted microscope with a $100 \times$ oil immersion objective (Olym- pus ACH, 1.25 N.A.), a piezo-driven microscope objective nanofocusing/scanning device (Physik Instrumente, PIFOC P-721.CDQ), a CCD camera (Pulnix TM_6710-CL) to capture images, parallel-light LED illumination, a motorized stage to control the height of the magnets above the flow cell (Physik Instrumente M-126.PD), and a motor to control the magnets' rotation (Physik Instrumente, C150). Flow cells were constructed from glass microscope cover slips with double-layer parafilm spacers and a nitrocellulose $(0.1 \%$ wt./vol. in amyl acetate) coated bottom surface. A syringe pump (Cole-Parmer) was used for buffer exchange in the flow cell. The setup is controlled by a Dell Precision T5400 workstation (Dell) and software custom written in Labview 8.6. Further details of this setup were reported previously. ${ }^{3,12,15,22}$ Experiments used either a conventional magnet configuration (referred to as "conventional MT") with a pair of $5 \mathrm{~mm} \times 5 \mathrm{~mm} \times 5 \mathrm{~mm}$ magnets (Supermagnete, W-05-N50-G) in horizontal configuration with an iron yoke ${ }^{12}$ or a cylindrical permanent magnet with a central aperture (Supermagnete, R-06-02-02-G) and an attached side magnet (Supermagnete, S-04-07-N) in the geometry described previously ${ }^{15}$ (referred to as "MTT"). CCD images are either analyzed in real time or recorded at frequencies up to $100 \mathrm{~Hz}$. The tracking software is implemented in Labview 8.6 and is available from the authors upon request.

\section{B. Beads, DNA constructs, and buffer conditions}

Experiments were carried out in phosphate buffered saline (PBS; $137 \mathrm{mM} \mathrm{NaCl}, 2.7 \mathrm{mM} \mathrm{KCl}, 10 \mathrm{mM}$ phosphate buffer, pH 7.4; Sigma) supplemented with $100 \mu \mathrm{g} / \mathrm{ml}$ BSA, $0.1 \%$ Tween, and $5 \mathrm{mM}$ sodium azide (PBS+). Prior to the measurements, $1.5 \mu \mathrm{m}$ radius non-magnetic latex beads (Life Sciences) were aspecifically attached to the bottom surface of the flow cell by incubation in phosphate buffered saline (PBS; Sigma) buffer for $30 \mathrm{~min}$ to act as reference beads. In addition, the bottom surface was functionalized by incubation with $100 \mu \mathrm{g} / \mathrm{ml}$ anti-digoxigenin (Roche) in PBS for $30 \mathrm{~min}$, to provide for DNA attachment, and passivated by incubation for $30 \mathrm{~min}$ with $2 \mathrm{mg} / \mathrm{ml}$ bovine serum albumin (BSA; Sigma). Experiments with tethered beads employed $7.9 \mathrm{kbp}$ DNA constructs ${ }^{15}$ ligated at the ends to $\approx 600$ bp DNA PCR fragments that were functionalized with multiple biotin and digoxigenin groups, respectively. The DNA molecules were first attached to streptavidin-coated $1.4 \mu \mathrm{m}$ radius superparamagnetic M270 beads (Invitrogen) by incubation in PBS buffer for 30-60 min. Subsequently, the DNA tethered superparamagnetic beads were incubated in the flow cell for $30 \mathrm{~min}$ in PBS buffer to allow for DNA attachment to the anti-digoxigenin coated surface. Unattached beads were removed by flushing with PBS+ buffer. Finally, biotin-labeled $0.5 \mu \mathrm{m}$ radius Fluosphere microspheres (Invitrogen) were stochastically attached to the M270 superparamagnetic beads to act as fiducial markers by incubation in the flow cell for 30-60 min in PBS+ buffer. 


\section{TRACKING ALGORITHMS}

\section{A. Overview of the tracking protocol}

Our tracking protocol is designed to follow the $\mathrm{x}, \mathrm{y}$, and $\mathrm{z}$ positions and rotation angle $\theta$ about the z-axis of tethered superparamagnetic beads from CCD images. In typical experiments, the tether consists of double-stranded DNA bearing multiple attachment points at both ends (Fig. 1(a)). We attach small, non-magnetic, marker beads to the larger superparamagnetic beads to provide well-visible fiducial markers that facilitate rotational tracking (Fig. 1(a); Sec. II).

The use of small fiducial marker beads is convenient and is motivated by the following considerations. While in principle intrinsic irregularities in the magnetic beads themselves can be employed for rotational tracking, ${ }^{23}$ in practice the superparamagnetic beads typically employed in MT measurements show only very minor deviations from spherical symmetry, making this approach less robust. This is particularly so in the case of the out-of-focus images advantageously employed for z-tracking. ${ }^{19,24}$ Another related approach to rotational tracking is the use of dumbbells of equally sized beads. ${ }^{16,20,25}$ Using dumbbells is less robust than using smaller fiducial marker beads if simultaneous ztracking over a significant focal range is required. In addition, if both beads of the dumbbell are magnetic, the analysis of the stretching force and the tether geometry is complicated compared to single-magnetic bead tethers.

A flow-chart diagramming the main steps in our tracking procedure is shown in Fig. 1(b). Our general strategy is to first determine the in-plane position ( $\mathrm{x}, \mathrm{y})$ of the tethered magnetic bead. To reliably find the (x,y)-position of the bead, a tracking protocol is needed that is minimally disturbed by the fiducial marker. We found that a tracker based on convolution with kernel images that exhibit periodic radial ring patterns filters out the perturbative effect of the fiducial marker (see Sec. III B). Using the tracked ( $\mathrm{x}, \mathrm{y})$-position, both the inplane rotation angle $(\theta)$ and the relative out-of-plane position (z) can be tracked using separate tracking modules (see Sec. III C, and Sec. III D).

\section{B. Tracking of the transverse position $(x, y)$}

Our kernel-based (x,y)-tracking is performed as follows. From camera-acquired microscope images, regions-ofinterest (ROIs) of size $\mathrm{N} \times \mathrm{N}$ pixels are selected, each consisting of an approximately centered defocused bead. This bead image is separately convoluted with three $\mathrm{N} \times \mathrm{N}$ pixelsized kernel images displaying similar centered ring-shaped patterns (Fig. 1(c)). The ring pattern of successive kernel images is shifted by one third of a period. The kernel images $\mathrm{K}(\mathrm{r})$ are numerically generated using:

$$
\mathrm{K}(\mathrm{r})=\mathrm{K}_{0} \cdot \mathrm{e}^{-r / r_{0}} \cdot \sin \left(\frac{\mathrm{r}}{\lambda}+\frac{2 \pi \mathrm{p}}{3}\right),
$$

where $\mathrm{K}_{0}$ is a proportionality constant, $\mathrm{r}$ is the distance from the image center, $r_{0}$ is a decay length, $\lambda$ is the fringe spacing (these last three quantities are all in units of pixel size), and $\mathrm{p}$ runs from 1 to 3 . Typical values for the parameters used in the tracking algorithm are given in Table I. To efficiently com-
TABLE I. Overview of parameters used in the tracking algorithm.

\begin{tabular}{lccc}
\hline \hline Parameter & Symbol & Typical value $^{\mathrm{a}}$ & Units \\
\hline Decay length & $\mathrm{r}_{0}$ & 65 & Pixel \\
Fringe spacing & $\lambda$ & 16 & Pixel \\
Proportionality constant & $\mathrm{K}_{0}$ & 1 & Pixel \\
Points per degree & $\mathrm{p}_{\mathrm{d}}$ & $5-20$ & Unitless \\
Points per radius & $\mathrm{p}_{\mathrm{r}}$ & 1 & Unitless \\
Inner radius (for radial tracking) & $\mathrm{r}_{\min }$ & 10 & Pixel \\
Outer radius (for radial tracking) & $\mathrm{r}_{\max }$ & 25 & Pixel \\
\hline \hline
\end{tabular}

${ }^{a}$ We report typical values that we found to enable robust tracking of $2.8 \mu \mathrm{m}$ diameter beads with $1.0 \mu \mathrm{m}$ fiducial markers.

pute the convolution between the bead image and the kernel images, we multiply the respective images in Fourier space and transform the results back to real space. The three correlation maps (Fig. 1(c), rightmost panels) will exhibit one global maximum $\left(\mathrm{x}_{\max }, \mathrm{y}_{\max }, \mathrm{p}_{\max }\right)$, indicating the best-fitting kernel image. These coordinates $\left(\mathrm{x}_{\max }, \mathrm{y}_{\max }\right)$ localize the bead center with single pixel resolution $(\approx 100 \mathrm{~nm}$ in our experimental configuration). To achieve a resolution of a few nanometers, a sub-pixel fitting step is performed by taking 5-point cross sections around the maximum of the correlation map along the $\mathrm{x}$ - and $\mathrm{y}$-directions. These cross sections are then subjected to a parabolic fit, whereby the sub-pixel location of the resulting maximum yields the final $(\mathrm{x}, \mathrm{y})$-coordinates. For outof-focus images, the kernel-based tracking amounts to spatial filtering and effectively locates the symmetry center of the main bead (Fig. 1(c)). Typically, the resulting (x, y)-position shifts only slightly in the direction of the fiducial marker (see Sec. IV A).

\section{Tracking of the rotation angle $(\theta)$}

Having determined the $(x, y)$-position of the bead, the CCD image can be further analyzed to track the in-plane rotation angle by effectively following the asymmetry induced by the marker bead(s). To do so, we compute an angular intensity profile $\overline{\mathbf{S}}(\theta)$ that represents the average intensity at every angle $\theta$, where the averaging is performed over the radial coordinate. To construct $\overline{\mathbf{S}}(\theta)$, the image of the bead together with its fiducial marker is first overlaid with a polar coordinate grid $(r, \theta)$ whose origin is located at the bead's center as determined via (x, y)-tracking (Fig. 2(a)). The angular coordinate has a range of $360^{\circ}$ that is segmented into $360 \cdot \mathrm{p}_{\mathrm{d}}$ steps, where $p_{d}$ is defined as the number of points per degree. The radial coordinate $r$ ranges between $r_{\min }$ and $r_{\max }$ with increments of $p_{r}$ per pixel unit. Here, we define one pixel unit as the horizontal (x) distance between two pixels.

Next, since points $(r, \theta)$ in the polar coordinate grid will not generally map onto integer-pixel $(i, j)$ coordinates, a proper estimate of the intensity $S_{\text {interpolated }}(r, \theta)$ for a given pair of polar coordinates $(r, \theta)$ relies on a series of interpolation steps involving the intensities of the four nearestneighbor pixels (Fig. 2(b)): two interpolation steps involving pixels spaced along the $\mathrm{x}$-coordinate and one interpolation step along the $y$-coordinate. Thus, we estimate the intensity at the point $(\mathrm{r}, \theta)$ by first interpolating along the $\mathrm{x}$-coordinate between the intensities $S_{00}$ and $S_{10}$ of the pixels $(0,0)$ and 

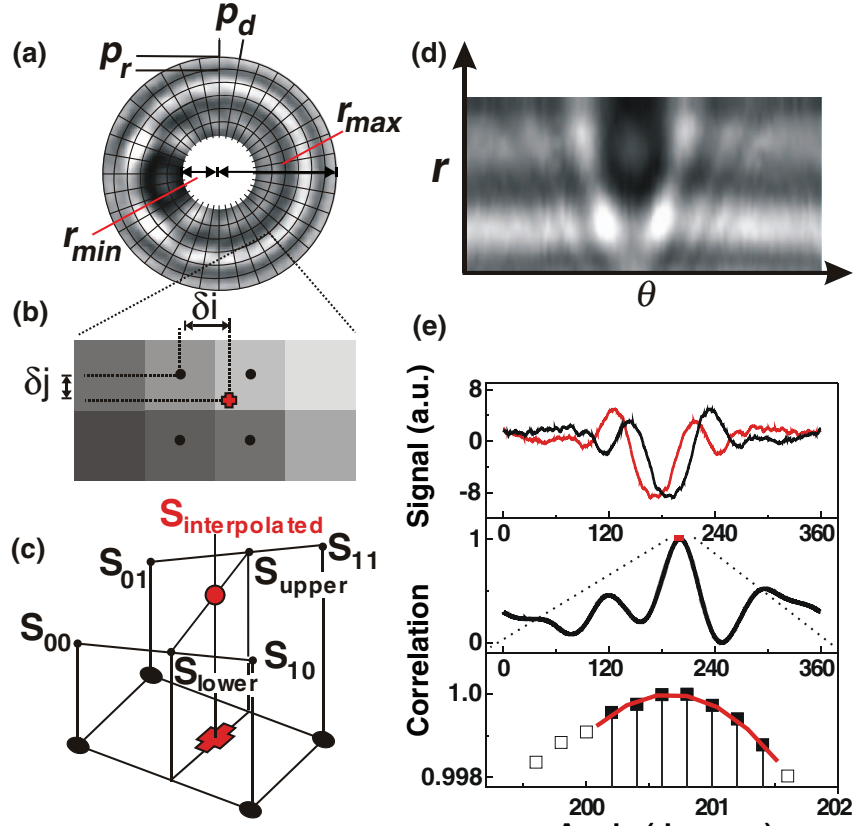

(e)

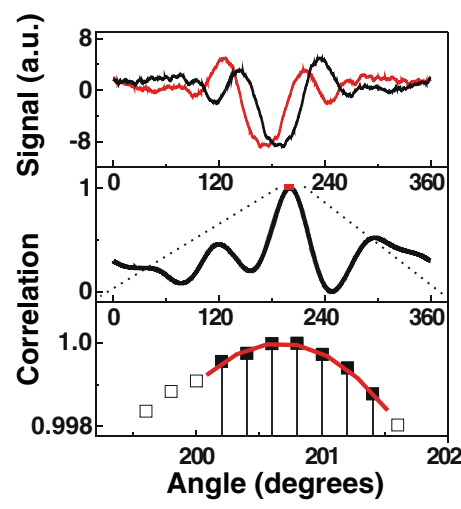

FIG. 2. (Color online) Image analysis for rotational tracking. (a) Image of a bead carrying a fiducial marker, cropped to the area that is employed for the rotational tracking. A bead-centered polar grid is overlayed. (b-c) Schematic closeup on individual pixels illustrating the interpolation step used to transform the pixel intensities of four neighboring pixels $S_{00}, S_{10}, S_{01}, S_{11}$ into an interpolated intensity value in polar coordinates $S_{\text {interpolated. }}$. (b) Pixel intensities are shown in grey scale and the pixel centers $S_{00}, \ldots, S_{11}$ are indicated by dots, the position of the interpolated intensity is indicated by a cross. (c) The pixel intensities are indicated by the height of the wireframe bars. (d) Radialpolar map of the selected image, i.e., pixel intensities of the image in (a) converted to polar $(\mathrm{r}, \theta)$ coordinates. (e) Analysis of the one-dimensional angular image signature to determine an angular position. (Upper panel) Summing of radial intensities from panel (d) yields an angular signature $\bar{S}(\theta)$ (darker curve). The mirror image of the radial signature is shown as the lighter curve. (Middle panel) Cross-correlation curve of the radial signature with its mirror image. (Lower panel) Closeup on the global maximum of the correlation curve and schematic of sub-pixel interpolation step to yield a final angular position.

$(1,0)$ :

$$
\mathrm{S}_{\text {lower }}=\mathrm{S}_{00}+\left(\frac{\mathrm{S}_{10}-\mathrm{S}_{00}}{\mathrm{~L}}\right) \delta_{i},
$$

where $\mathrm{L}$ is the distance between these two pixels (in pixel units, L equals 1 ) and $\delta_{\mathrm{i}}$ is the value of the x-projection of the point $(\mathrm{r}, \theta)$ relative to the "left-most" pair of pixels $(0,0)$ and $(0,1)$ located nearest to the $y$-axis. The value of $S_{\text {lower }}$ is shown graphically in Fig. 2(c). Analogously, we perform a second interpolation along the $\mathrm{x}$-coordinate using the intensities of the two pixels $(0,1)$ and $(1,1)$ to yield $S_{\text {upper }}$ (Fig. 2(c)). We then perform an similar interpolation along the y-coordinate using $\mathrm{S}_{\text {upper }}, \mathrm{S}_{\text {lower }}$, and $\delta_{\mathrm{j}}$ (the value of the y-projection of the point $(\mathrm{r}, \theta)$ relative to the "bottom" pair of pixels $(0,0)$ and $(1,0))$ to yield a best estimate for $S_{\text {interpolated }}(r, \theta)$ (Fig. 2(c)):

$$
\mathrm{S}_{\text {interpolated }}(\mathrm{r}, \theta)=\mathrm{S}_{\text {lower }}+\left(\frac{\mathrm{S}_{\text {upper }}-\mathrm{S}_{\text {lower }}}{\mathrm{L}}\right) \delta_{\mathrm{j}} .
$$

Using this two-dimensional interpolation, we generate a "unfolded" polar map (Fig. 2(d)). Summing this polar map over the radial coordinate $\mathrm{r}$ then yields the desired one- dimensional angular intensity profile $\overline{\mathbf{S}}(\theta)$ (Fig. 2(e), upper panel, red curve). As expected, $\overline{\mathbf{S}}(\theta)$ exhibits the largest amplitude variations in the angular region in which the small marker bead is located (Fig. 2(e), upper panel).

The angular position of the small marker bead can now be computed by performing a one-dimensional cross correlation of $\overline{\mathbf{S}}(\theta)$ with its own mirror image $\overline{\mathbf{S}}(360-\theta)$ (Fig. 2(e), upper panel). The corresponding correlation curve exhibits a pronounced maximum that is readily detected (Fig. 2(e), central panel). Note that a center-symmetric pattern, for example, a marker located in the center of the unfolded pattern midway between $0^{\circ}$ and $360^{\circ}$ as in Fig. 2(d), would yield a correlation peak centered at $0^{\circ}$. To simplify the subsequent fitting (see below), the curve halves are swapped such that the resulting correlation peak centers at $180^{\circ}$ instead. Any deviation from a center-symmetric pattern moves the correlation peak away from this defined mid-point. We note that the maximum in the correlation curve shifts twice as fast as any imposed rotation, yielding tracked angles that exhibit a periodicity of $\pi$ (as opposed to the correct $2 \pi$ ). Additionally, we note that our correlation algorithm does not make any use of previously tracked positions, which improves its robustness.

Analogously to the (x,y)-tracking procedure described above, the precise location of the correlation maximum is determined by a 5-point parabolic fit (Fig. 2(e), lower panel). The resulting angular coordinate is scaled by $\mathrm{p}_{\mathrm{d}} / 2$ to yield an angle in degrees. The expected half-turn periodicity (see above) is corrected a posteriori by removing angular jumps with values sufficiently close to $\pi$. Straightforward discrimination between instantaneous phase jumps and physical signal features is justified as the time response along the rotational axis of the bead-tether construct is typically significantly lower than the time interval between successive acquisition frames.

\section{Tracking of the height (z)}

The last component in the tracking algorithm involves tracking of the height $\mathrm{z}$ of the tethered magnetic bead above the surface. Height tracking is performed by monitoring the changes in the defocused ring pattern when the imaged bead shifts in height relative to the focal plane. To do so, we employ averaged radial intensity profiles $\overline{\mathrm{S}}(\mathrm{r})$. To construct the $\overline{\mathrm{S}}(\mathrm{r})$ profiles, we first define a radial axis $\mathrm{r}$ emanating from the previously determined center position $(\mathrm{x}, \mathrm{y})$. This radial axis is then segmented into bins that typically have a size equal to one pixel unit. To obtain the average radial intensities, we first loop through all pixels (i, j) of the image and obtain two arrays: one of total pixel intensities $\mathrm{S}_{\mathrm{SUM}}(\mathrm{r})$ per radial bin, and one of total pixel counts $\mathrm{N}(\mathrm{r})$ per radial bin. By combining these two radial arrays, a radial profile can be computed according to:

$$
\bar{S}(r)=\frac{S_{\text {SUM }}(r)}{N(r)} \text {. }
$$

As in the case of the computation of the one-dimensional angular intensity profile $\overline{\mathbf{S}}(\theta)$, proper computation of the 
intensities $\overline{\mathrm{S}}(\mathrm{r})$ benefits from interpolation. We note that the radius associated with a pixel located at coordinate index $(\mathrm{i}, \mathrm{j})$ is $r_{i j}=\sqrt{\left(x-x_{i j}\right)^{2}+\left(y-y_{i j}\right)^{2}}$, where $\left(\mathrm{x}_{\mathrm{ij}}, \mathrm{y}_{\mathrm{ij}}\right)$ are the associated pixel coordinates and $(\mathrm{x}, \mathrm{y})$ represent the previously determined center position of the bead pattern. While for the purposes of computing $\mathrm{S}_{\mathrm{SUM}}(\mathrm{r})$ and $\mathrm{N}(\mathrm{r})$ this radius could simply be rounded to the nearest bin value, such rounding causes strong discretization effects in the final tracking results (data not shown). Therefore, we instead partition the pixel intensity $S_{i j}$ between the two nearest radial bins $r_{\text {lower }}$ and $r_{\text {upper }}$, again relying on a simple interpolation scheme: $\mathrm{S}_{\mathrm{SUM}}\left(\mathrm{r}_{\text {lower }}\right)$ is increased by $\left(\mathrm{r}_{\text {upper }}-\mathrm{r}_{\mathrm{ij}}\right) /\left(\mathrm{r}_{\text {upper }}-\mathrm{r}_{\text {lower }}\right) \cdot \mathrm{S}_{\mathrm{ij}}$, and the associated count $\mathrm{N}\left(\mathrm{r}_{\text {lower }}\right)$ is increased by a "fractional count" equal to $\left(r_{\text {upper }}-r_{i j}\right) /\left(r_{\text {upper }}-r_{\text {lower }}\right)$. The values of $\mathrm{S}_{\mathrm{SUM}}\left(\mathrm{r}_{\text {upper }}\right)$ and $\mathrm{N}\left(\mathrm{r}_{\text {upper }}\right)$ are similarly computed. Here, the suffixes "lower" and "upper" refer to the nearest bins with lower and higher radii compared to the radius $\mathrm{r}_{\mathrm{ij}}$, respectively. After processing all image pixels and in this manner filling both radial arrays, we compute the average radial intensity according to Eq. (4).

For each tethered magnetic bead, a stack of such radial signatures is recorded as a function of relative focus height by displacing the objective in fixed z-increments at the start of an experiment, resulting in a look-up-table (LUT). To track the z-coordinate during measurements of tethered magnetic beads, the summed squared difference of an instantaneous radial signature $\bar{S}(r)$ and each row of the LUT results in an error signal:

$$
\varepsilon(\mathrm{z})=\frac{1}{\mathrm{~N}} \sum_{\mathrm{r}}[\operatorname{LUT}(\mathrm{z}, \mathrm{r})-\overline{\mathrm{S}}(\mathrm{r})]^{2} .
$$

The minimum of the function in Eq. (5) designates the focal plane that best fits the actual image. Analogously to the procedures employed in tracking $(\mathrm{x}, \mathrm{y})$ and $\theta$, a more precise, sub-plane height estimate is obtained by fitting a 5-point parabolic fit to this error signal. ${ }^{19}$

\section{RESULTS}

\section{A. Evaluation of the tracking algorithms using simulated images}

To evaluate the performance of our position and angular tracking algorithms, we first test them on simulated bead images and then on experimental data. To evaluate the tracker relative to an exact reference, we generated artificial bead images closely resembling those obtained via bright-field microscopy. As the positional and angular coordinates of such simulated bead images are known exactly, both tracking precision and accuracy can be evaluated as the difference between simulated positions and tracked results. Since our primary interest is rotational tracking, we focus on a non-translating, purely rotating pattern for which we evaluate the tracked rotational coordinate as well as the $\mathrm{x}$-position. The evaluation is performed in the presence of varying degrees of added noise to simulate the effects of camera noise.

To simulate bead images that have both an overall pattern and a fiducial marker location that closely resembles experimentally observed images (Fig. 1), we assign the main bead a radius of $R_{\text {main }}$ and the smaller fiducial bead a radius $R_{\text {fid }}$ (a)

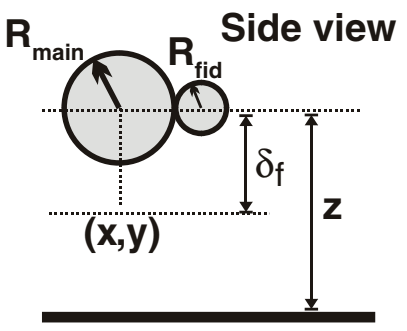

(b) Look-up table

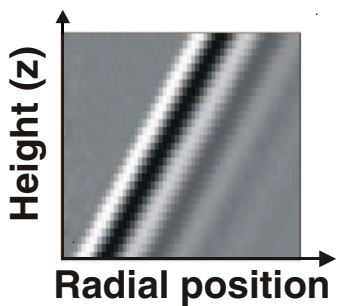

(c)

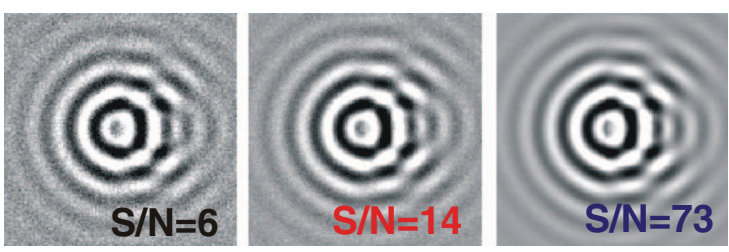

(d)
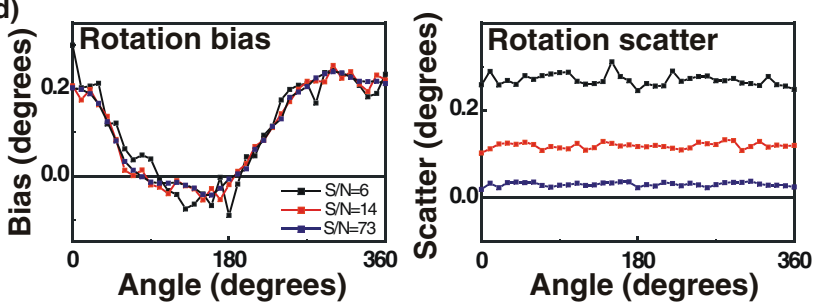

(e)
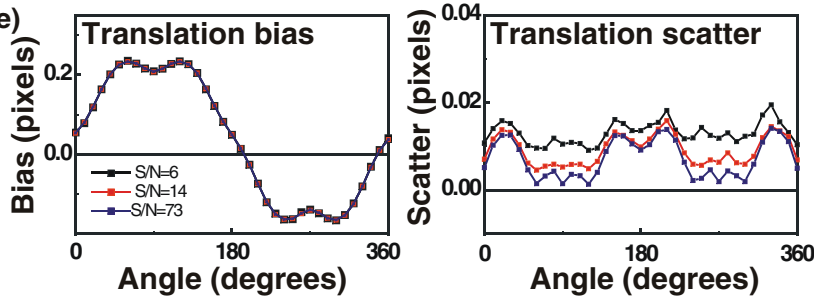

FIG. 3. (Color online) Evaluation of the tracker performance using simulated images. (a) Simulation geometry, with a main and a fiducial bead, including the relative position in the focal plane. (b) Radial fringe pattern as a function of height, i.e., the artificial look-up-table for the simulated images. (c) Simulated images for three different signal-to-noise levels. (d) Bias (left panel) and scatter (right panel) in the rotation angle for tracking results compared to preset pattern positions for the three signal-to-noise noise levels shown in panel (c). (e) Analogous plots for the tracking bias (left panel) and scatter (right panel) in the x-position.

(attached at a vertical angle $\phi$; Fig. 3(a)). For both beads, we generate separate patterns defocused from each geometrical center according to:

$$
\begin{aligned}
S(i, j)= & A \cdot\left(\frac{R_{\text {main or fid }}}{R_{\text {main }}}\right)^{3} \cdot \sin \left(2 \pi \frac{r-f_{0}}{\lambda}\right) \cdot \mathrm{e}^{\frac{-\left(r-f_{0}\right)}{k_{0}}} \\
& \cdot\left(1-\mathrm{e}^{\frac{-\left(r-f_{0}\right)}{5 \lambda}}\right),
\end{aligned}
$$

where $r$ is the respective distance from the main or fiducial bead center, $\mathrm{A}$ is a proportionality constant, and the ratio of the cubed radii (i.e., the scattering volume) is used to scale the intensity of the bead images. Additionally, the parameters $\lambda$ and $\mathrm{k}_{0}$ are representative values for the fringe spacing and the pattern decay length, and $f_{0}$ indicates the position of the focal plane (thus introducing a simple expanding fringe pattern upon defocusing; Fig. 3(b)). Patterns generated in this manner are superimposed on an image containing a background intensity B as well as a Gaussian-distributed noise with a standard deviation of $\sigma$ per pixel. For comparison with experimental 
TABLE II. Overview of parameters used to generate the artificial image employed for testing the tracking algorithm.

\begin{tabular}{lccl}
\hline \hline Parameter & Symbol & Typical value & Units \\
\hline Image size & $\ldots$ & 140 & Pixel \\
Image depth & $\ldots$ & 255 & Grey level \\
Background level & $\mathrm{B}$ & 100 & Grey level \\
Noise level & $\sigma$ & $0-50$ & Grey level \\
Proportionality constant & $\mathrm{A}$ & 75 & Unitless \\
Main bead radius & $\mathrm{R}_{\text {main }}$ & 20 & Pixel \\
Fiducial bead radius & $\mathrm{R}_{\text {fid }}$ & 15 & Pixel \\
Fiducial bead vertical attachment angle & $\phi$ & 45 & Degrees \\
Defocus & $\mathrm{f}_{0}$ & $1-100$ & Unitless \\
Fringe spacing & $\lambda$ & 15 & Pixel \\
Pattern decay & $\mathrm{k}_{0}$ & 45 & Pixel \\
\hline \hline
\end{tabular}

images, we define an empirical signal-to-noise ratio $\mathrm{SN}_{\mathrm{e}}$ :

$$
\mathrm{SN}_{\mathrm{e}}=\frac{\sqrt{\mathrm{S}_{\text {range }}^{2}-\Delta^{2}}}{\Delta} .
$$

We take the range of the image intensity $S_{\text {range }}$ as a measure for signal level, and we estimate a $95 \%$ confidence interval $\Delta$ for the noise via the standard deviation of the intensity at the image's edges. Using this approach, we find that typical experimental 8-bit images exhibit a $\mathrm{SN}_{\mathrm{e}}$ ratio of between 20 and 40 (Figs. 1 and 3(c)).

We next applied our tracking algorithm to series of simulated images and compared the results with the exactly known input positions (Table II and Figs. 3(d) and 3(e)). The difference between input and tracked positions yields the tracking error per image. In general, a tracking error consists of two components: the accuracy, i.e., the systematic deviation or "bias" and the precision or "scatter," i.e., the statistical variation due to noise in the images. The latter is simply evaluated for a given pattern position by a series of images that differ only in the statistical noise added. For such a series, the average deviation from the input positions yields the bias, while the standard deviation yields the scatter. By performing stepwise position changes, bias and scatter can thus be systematically evaluated for any type of motion.

We evaluated the bias and scatter for simple rotational motion in the absence of translation, using three representative noise levels (Fig. 3(c)). We first evaluated the bias error (Figs. 3(d) and 3(e), left panels) and the scatter error (Figs. 3(d) and 3(e), right panels) for the angular coordinate. We observe a maximum structural deviation from the correct angle of approximately $0.2^{\circ}$ (Fig. 3(d), left panel) and we observe a $0.1^{\circ}$ scatter at a $\mathrm{S} / \mathrm{N}$ ratio of 14 (Fig. 3(d), right panel), which is at the lower range of experimental values. We repeat the evaluation of the bias error (Fig. 3(e), left panel) and the scatter error (Fig. 3(e), right panel) for the x-coordinate. As motion in the $\mathrm{x}$-direction was absent in the simulation, this analysis reports on the crosstalk between angular and translational motion axes. The translational axes exhibit a non-zero scatter of a few hundredths of a pixel, which amounts to a few nanometers in comparable experimental images (Fig. 3(e), right panel). The magnitude of the translational scatter appears fairly independent of the type of motion performed, as can be expected since it stems mainly from camera noise. For the bias, we observe a deviation in the apparent $\mathrm{x}$ position of at most $\sim 0.2$ pixels (Fig. 3(e), left panel; identical results are obtained for the y-coordinate). This confirms that the presence of the marker does not strongly bias the position tracking, and that only very slight crosstalk exists between the angular and translational coordinates. Indeed, this level of bias corresponds to a few tens of nm given typical magnifications and camera pixel sizes and is to be expected from the residual perturbation of the pattern associated with a fiducial marker. We note in many experimental situations only integer number of magnet turns are considered: if the resulting number of bead rotations are also nearly integer, the bias in both position and angle is negligible. Overall, the results for simulated images indicate that our tracking algorithm is sufficiently robust against the perturbation introduced by the fiducial marker beads and achieves a precision of $\sim 0.1^{\circ}$ and $\sim 1-2 \mathrm{~nm}$ for realistic $\mathrm{S} / \mathrm{N}$ ratios.

\section{B. Experimental results for surface-immobilized beads}

To establish the performance of our tracking protocol under experimental conditions, we recorded times trace of CCD images in our magnetic tweezers setup. First, we recorded traces of fixed beads, stuck aspecifically to the flow cell surface (Fig. 4). Tracking fixed beads provides a good way to evaluate the realistic experimental tracking precision as the expected position and angular signal are constant. ${ }^{26-28}$ Any apparent motion will include both inaccuracies of the tracking algorithm and other experimental errors, such as mechanical drift, optical aberrations, possible residual motion of the beads, etc. To stay close to the experimental situation typical of measurements for DNA-tethered beads, we select a pair of fixed beads that is identical to those used for tethered bead measurements, comprising of a $3.0 \mu \mathrm{m}$ diameter polystyrene reference bead and a $2.8 \mu \mathrm{m}$ diameter superparamagnetic bead carrying a $1.0 \mu \mathrm{m}$ diameter fiducial marker bead. Series of CCD images were recorded at $30 \mathrm{~Hz}$ at different focus settings, with the bead pair in focus (Fig. 4(a)), $\sim 2.5 \mu \mathrm{m}$ below focus (Fig. 4(b)), $\sim 4.5 \mu \mathrm{m}$ below focus (Fig. 4(c)), and $\sim 7.5 \mu \mathrm{m}$ below focus (Fig. 4(d)). Representative images of the "signal" bead carrying a fiducial marker at the corresponding focus settings are shown as insets in Fig. 4. The x, y, and z-positions are obtained by subtracting the reference bead position from the position of the "signal" bead. Importantly, to test the robustness of the algorithm, all traces were tracked with the same settings for the tracking algorithm (Table I). The observed fluctuations in the $(\mathrm{x}, \mathrm{y}, \mathrm{z})$-positions are approximately Gaussian (Figs. 4(a)-4(d), histograms in the right panels) and the standard deviations of the fluctuations provide a measure for the precision of the tracker. For beads positioned 2.5-7.5 $\mu \mathrm{m}$ below focus, we find similar precision in $\mathrm{x}, \mathrm{y}$, and $\mathrm{z}$ with $\sigma_{\mathrm{x}}$ $\approx \sigma_{\mathrm{y}} \approx \sigma_{\mathrm{z}} \approx 1.5 \mathrm{~nm}$. If the beads are in focus, the precision deteriorates, in particular for the z-tracking (Fig. 4(a)). This is to be expected, since the $\mathrm{z}$-tracking relies on the diffraction ring pattern, which is much less pronounced if the beads are 
(a)

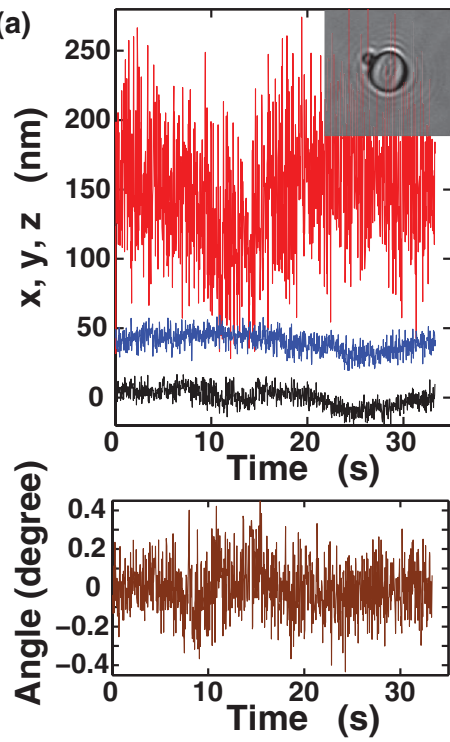

(b)
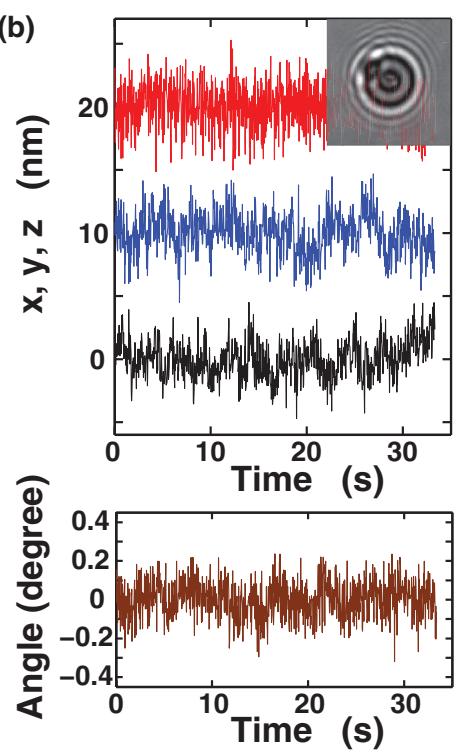
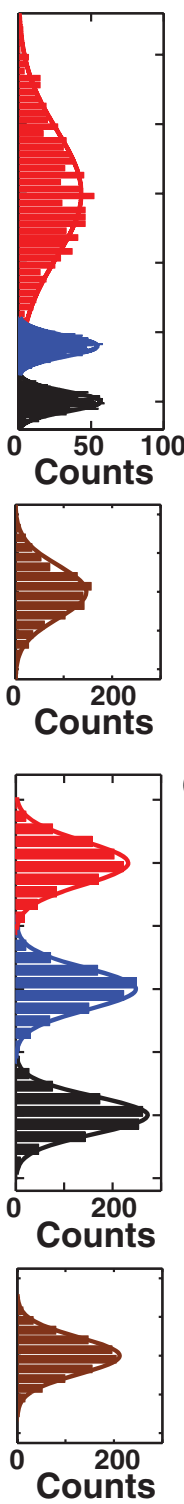

(c)
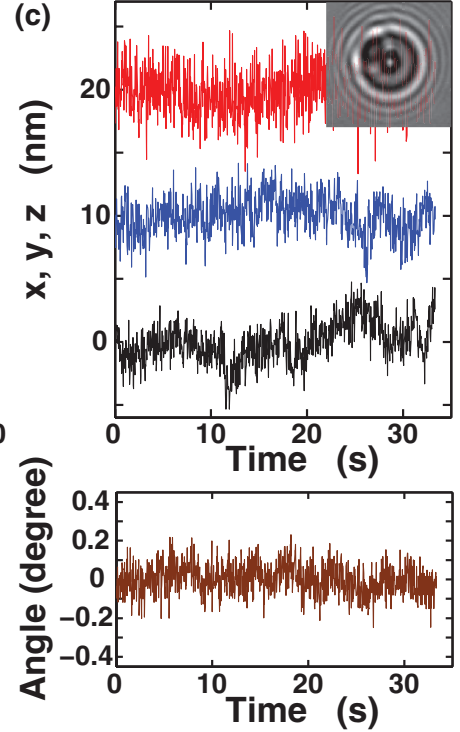

(d)
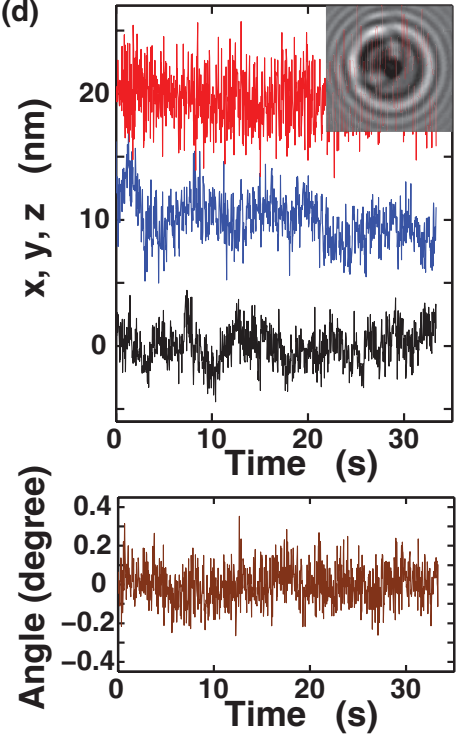
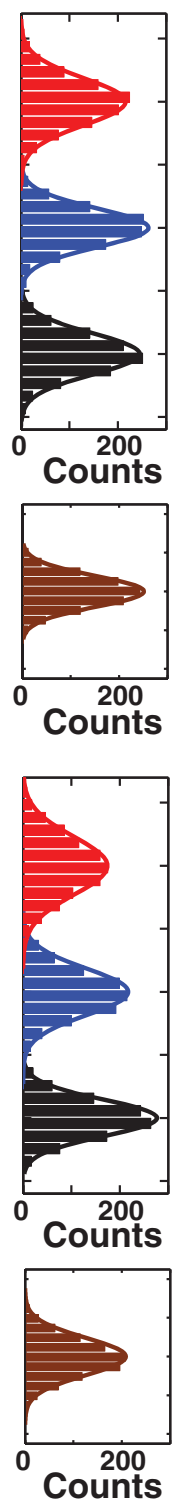

FIG. 4. (Color online) Angular and positional tracking for surface-immobilized beads at different distances with respect to the focal plane. We recorded time traces of camera images of two beads non-specifically attached to the flow cell surface. One of the beads is a $2.8 \mu \mathrm{m}$ diameter bead with a $1.0 \mu \mathrm{m}$ fiducial marker bead attached, the other is a $3.0 \mu \mathrm{m}$ reference bead, identical to the situation used for tracking tethered beads (Figs. 5 and 6). Traces of the same beads were recorded at different focus settings, approximately in focus (a), $\sim 2.5 \mu \mathrm{m}$ out of focus (b), $\sim 4.5 \mu \mathrm{m}$ out of focus (c), and $\sim 7.5 \mu \mathrm{m}$ out of focus (d). Corresponding images of the bead carrying the fiducial marker are shown as insets in panels (a)-(d). In each panel, the results of (x, y, z)-position (bottom, middle, and top traces, respectively) and angular tracking (separate panels) are shown, with the traces offset for clarity. Histograms of the position and angle distributions are shown in the right sub-panels (same order and color code). Solid lines in these sub-panels are Gaussian fits. The standard deviations of the traces are $\sigma(\mathrm{x}, \mathrm{y}, \mathrm{z}, \theta)=(7.03 \mathrm{~nm}$, $\left.7.33 \mathrm{~nm}, 46.1 \mathrm{~nm}, 0.136^{\circ}\right)$ for panel (a), $\sigma(\mathrm{x}, \mathrm{y}, \mathrm{z}, \theta)=\left(1.47 \mathrm{~nm}, 1.61 \mathrm{~nm}, 1.72 \mathrm{~nm}, 0.094^{\circ}\right)$ for panel (b), $\sigma(\mathrm{x}, \mathrm{y}, \mathrm{z}, \theta)=\left(1.63 \mathrm{~nm}, 1.52 \mathrm{~nm}, 1.85 \mathrm{~nm}, 0.079^{\circ}\right)$ for panel c), and $\sigma(\mathrm{x}, \mathrm{y}, \mathrm{z}, \theta)=\left(1.44 \mathrm{~nm}, 1.84 \mathrm{~nm}, 2.29 \mathrm{~nm}, 0.096^{\circ}\right)$ for panel $\left.\mathrm{d}\right)$.

in focus. Similarly, the precision of the tracking routine suffers if the beads are too far out of focus; this trend is already discernible for the z-trace at $7.5 \mu \mathrm{m}$ out of focus (Fig. 4(d)).

The angular traces are obtained directly from analysis of the signal bead, without subtraction of a reference signal. Test measurements with a reference bead also carrying a fiducial marker for angular tracking showed no significant improvements from subtraction of the reference angle (data not shown). Analysis of the angle traces demonstrates a precision of $\sigma_{\theta} \approx 0.1^{\circ}$ in the focus range $2.5-7.5 \mu \mathrm{m}$ and only a slight deterioration of the angle tracking precision if the bead is in focus, which likely stems from the reduced precision of the $(\mathrm{x}, \mathrm{y})$-tracking.
Taken together, the results from tracking immobilized beads indicate a precision of $1-2 \mathrm{~nm}$ in $(\mathrm{x}, \mathrm{y}, \mathrm{z})$ and $0.1^{\circ}$ in angle over a wide range of focus settings. This level of precision is in line with the predictions from simulations (Fig. 3), since the signal-to-noise ratio (Eq. (7)) of our experimental images is $\approx 30$. Our position resolution is similar to that reported in other magnetic tweezers studies. ${ }^{19,26,28}$ We note that since our primary aim was to develop a reliable rotational tracking protocol, we did not particularly optimize our setup for position tracking precision, e.g., by optimizing the illumination, camera, or bead attachment protocol. The ability to track $(x, y, z)$ and angle over a $\mu \mathrm{m}$-range of focus settings is convenient for experiments where the length of the DNA tether changes, e.g., 
if the stretching force is adjusted or if the DNA is supercoiled during the experiment, processes that will typically give rise to $\sim \mu \mathrm{m}$ changes in tether length and therefore bead height.

\section{Experimental results for tethered beads and multi-bead tracking}

Having established the precision of our tracking protocol, we next tested the approach for DNA-tethered beads. We employed $7.9 \mathrm{kbp}$ DNA constructs with multiple attachment points at either end to tether $2.8 \mu \mathrm{m}$ diameter M270 superparamagnetic beads carrying $1.0 \mu \mathrm{m}$ diameter fiducial markers (see Sec. II). It is possible to have multiple tethered beads in one field of view of the camera (Fig. 5(a)) and our tracker can conveniently determine the $(\mathrm{x}, \mathrm{y}, \mathrm{z})$-position and rotation angle of multiple beads in separately defined regions of interest from recorded images. This kind of multi-bead tracking has the potential to significantly enhance the throughput of single molecule MT experiments. ${ }^{26,29,31}$ While our implementation of the tracking algorithm is suited for multi-bead tracking, we note, however, that our experimental protocol was not optimized to achieve a large number of beads in the field of view. If massively parallel multi-bead tracking is desired, (i) a lower magnification, (ii) a larger field of view camera, and (iii) a surface protocol optimized for a high-density of tethers should be employed in future experiments.

The traces of DNA-tethered beads stretched upwards by the magnetic field exerted by a pair of cubic magnets (the conventional magnet configuration, see Sec. II) reveal Brownian fluctuations in both the (x,y)-position and the angular coordinate. Traces of fluctuations in the $\mathrm{x}$-position are shown in Fig. 5(b), and similar excursions are recorded for the y-position (data not shown). As expected, the magnitude of these Brownian fluctuations, $\sigma_{\mathrm{x}} \approx 70 \mathrm{~nm}$ (Fig. 5(b)), is much larger than the apparent motion of surface immobilized beads used as a reference (Fig. 5(b), magenta trace) and related to the stretching force, ${ }^{2,3,5,13} \mathrm{~F} \approx 2 \mathrm{pN}$ in this case. Similar to the position fluctuations, the tethered beads undergo Brownian fluctuations in $\theta$ that are much larger than the apparent fluctuations observed for stuck beads (compare Fig. 5(c) to Fig. 4). The results shown in Fig. 5 and from a number of similar traces indicate that our tracking algorithm can reliably follow the (x, y, z)-positions and angular fluctuations of tethered beads. In particular, the tracking protocol is fairly insensitive to the exact attachment point of the fiducial marker bead (compare, e.g., the marker in the blue and red regions of interest in Fig. 5(a)), tolerates partially overlapping bead images (Fig. 5(a), red region of interest) provided the overlap is not too large, and is robust against deformations of the maker (Fig. 5(a), black region of interest; the marker consists of a small cluster of non-magnetic beads).

From the angular fluctuations of tethered beads we can additionally deduce the stiffness of the angular trap imposed by the magnets. Employing a conventional MT magnet geometry with a pair of magnets positioned $2 \mathrm{~mm}$ from the flow cell surface, the fluctuations have magnitude of $\sigma_{\theta}$ $\approx 0.9^{\circ}-1.5^{\circ}$ (Fig. 5(c)). For this magnet geometry, the magnetic field is aligned along the $\mathrm{x}$-axis, with a field strength $\mathrm{B}$ $\approx 23 \mathrm{mT}$, calculated from finite element simulations. ${ }^{12}$ The (a)

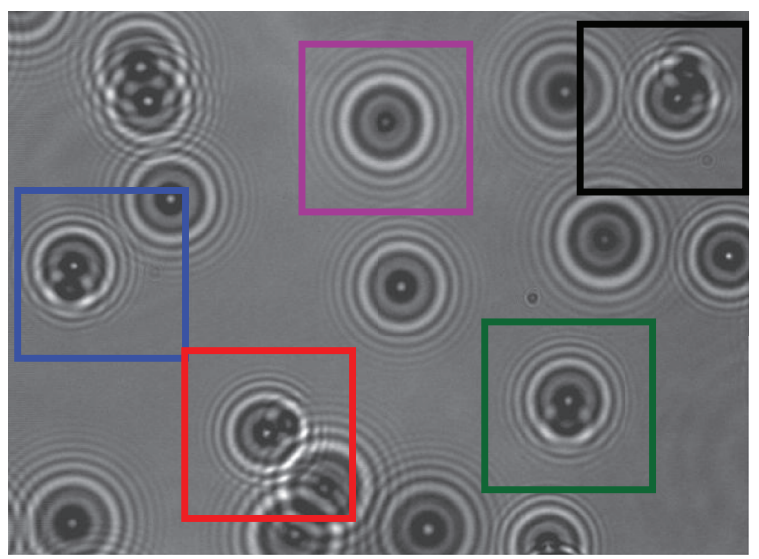

(b)

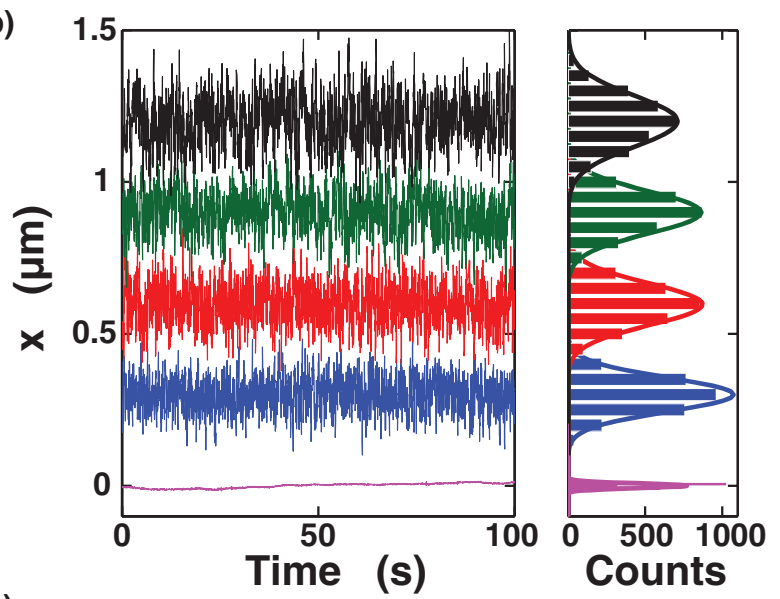

(c)

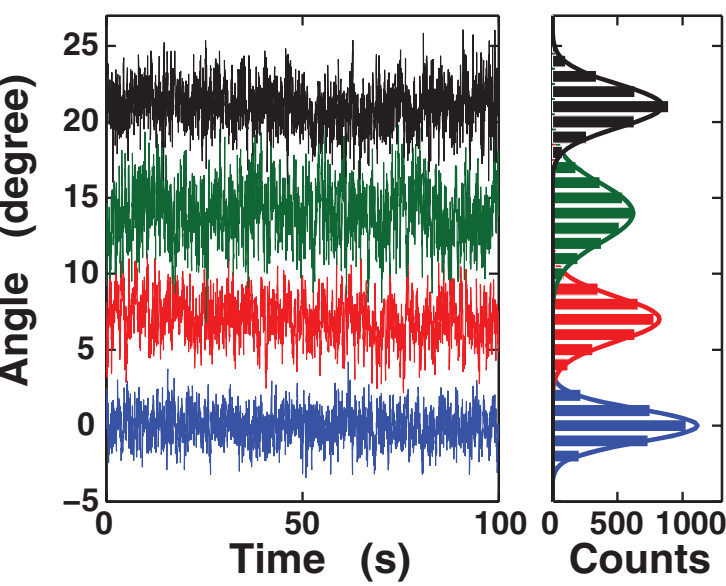

FIG. 5. (Color online) Angular and position tracking for multiple DNAtethered beads. (a) Field of view of the CCD camera. The measurement employed a $100 \times$ objective. At this magnification the total field of view is $60 \mu \mathrm{m}$ $\times 44 \mu \mathrm{m}$. The frames denote the regions of interest of 140 pixels $\times 140$ pixels that were selected to track individual beads. The bead in the top-center frame is a $3 \mu \mathrm{m}$ diameter Latex bead non-specifically stuck to the surface that was used as a reference bead. The beads in the other frames are $2.8 \mu \mathrm{m}$ diameter M270 superparamagnetic beads carrying one or multiple $1.0 \mu \mathrm{m}$ diameter marker beads, tethered by $7.9 \mathrm{kbp}$ DNA constructs to the flow cell surface. (b) Time traces and histograms of the $\mathrm{x}$-positions. The color code corresponds to the frames in panel (a); the bottom trace is that of the reference bead. Traces are offset for clarity. Solid lines in the right panel correspond to Gaussian fits. (c) Time traces and histograms of the rotation angles. The color code corresponds to the frames in panel (a); since the reference bead does not have a fiducial marker, we do not report an angle for that bead. Traces are offset for clarity. Solid lines in the right panel correspond to Gaussian fits.

magnetic field sets up an effective rotational trap constraining rotation about the z-axis. The stiffness of this rotational 
trap $\mathrm{k}_{\theta}$ can be determined from the variance of the rotational fluctuations $\sigma_{\theta}^{2}$ :

$$
\mathrm{k}_{\theta}=\frac{\mathrm{k}_{\mathrm{B}} \mathrm{T}}{\sigma_{\theta}^{2}} \text {, }
$$

where $\mathrm{k}_{\mathrm{B}}$ is the Boltzmann constant and $\mathrm{T}$ is the absolute temperature. Fluctuations with $\sigma_{\theta} \approx 1^{\circ}$ correspond to a torsional trap stiffness $\mathrm{k}_{\theta} \approx 13 \mathrm{pN} \mu \mathrm{m} / \mathrm{rad}$. Our measurement for M270 beads can be compared to two measurements for M280 beads reported in the literature. Klaue and Seidel report a value of $\mathrm{k}_{\theta}$ $\approx 20 \mathrm{pN} \mu \mathrm{m} / \mathrm{rad}$ at a field strength similar to our experiment from an indirect measurement analyzing the z-fluctuations. ${ }^{28}$ Janssen et al. propose a simple model for the rotational trap stiffness assuming a permanent component of the magnetization $\mathrm{m}_{0}$, where $\mathrm{k}_{\theta}=\mathrm{m}_{0} \mathrm{~B}$. These authors find $\mathrm{m}_{0}$ $\approx 10^{-15} \mathrm{~A} \mathrm{~m}^{2}$ for M280 beads. ${ }^{23}$ Their linear model implies $\mathrm{k}_{\theta} \approx 23 \mathrm{pN} \mu \mathrm{m} / \mathrm{rad}$ at $\mathrm{B} \approx 23 \mathrm{mT}$, in reasonable agreement with the measurement from the $\mathrm{z}$-fluctuations by Klaue and Seidel and within a factor of 2-4 of our measurements on M270 beads, suggesting that the M270 beads used in our experiments have a similar, yet somewhat smaller preferred axis compared to M280 beads.

\section{Tracking bead rotation and the torsional response of DNA}

Finally, we demonstrate the capability of our tracking protocol to accurately follow rotating beads and to detect shifts in angular distributions that enable the direct measurement of torque. DNA-tethered beads can be rotated about the $\mathrm{z}$-axis by rotating the permanent magnets of either conventional MT or MTT. Figure 6(a) shows an example trace of the tracked rotation angle of a $2.8 \mu \mathrm{m}$ diameter M270 bead carrying a $1.0 \mu \mathrm{m}$ diameter fiducial marker bead in the MTT. Initially, the magnets are static and the bead undergoes thermal fluctuations around its equilibrium angle position. Subsequently, the magnets are rotated counterclockwise (corresponding to positive rotation angle) by 10 turns. The rotation is readily discerned in the CCD images (Fig. 6(a), top inset) and accurately followed by the angular tracking protocol (Fig. 6(a), blue trace between $\sim 100$ and 200 s). After completing 10 turns, another plateau of thermal rotational fluctuation is recorded (Fig. 6(a), right inset). Subsequently, the magnets are rotated by 10 turns in the opposite (clockwise) direction, returning the tethered bead to its initial rotational state.

Systematically rotating the tether bead counterclockwise (clockwise) will overwind (underwind) the DNA tether, provided that the molecule is fully double-stranded and attached by multiple attachment points at both ends. Over- and underwinding DNA molecules in this fashion leads to the accumulation of torsional strain after a number of rotations. Due to this accumulation of torsional strain, over- or underwound molecules exert a restoring torque $\tau_{\text {DNA }}$ on the bead. The restoring torque can be measured by observing shifts in the mean angle position of the plateaus, $\left\langle\theta-\theta_{0}\right\rangle$, and calibrating the trap stiffness of the magnetic trap $\mathrm{k}_{\theta}$ from thermal
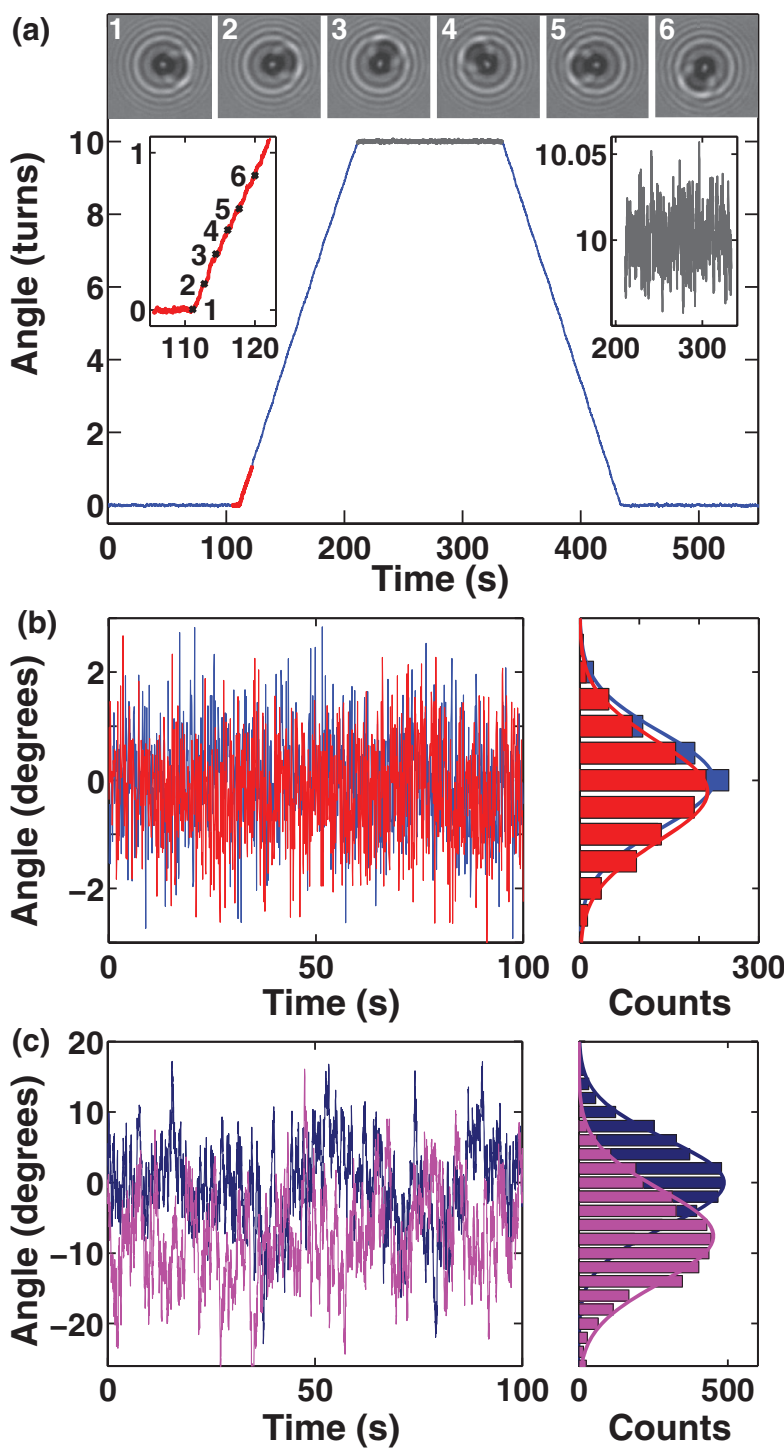

FIG. 6. (Color online) Angle tracking of DNA-tethered beads to detect bead rotation and torque. (a) Angle trace of a DNA tethered bead manipulated in the magnetic torque tweezers. Initially, the bead undergoes equilibrium fluctuations; at $\sim 110 \mathrm{~s}$, the magnets are rotated 10 turns at $0.1 \mathrm{~Hz}$. The rotation of the bead is followed by the angular tracking algorithm. The left inset shows a closeup of the angle trace during the first full turn. Selected frames are marked in the trace and numbered; the corresponding camera images of the bead are shown in the inset on top. After the initial 10 turns, a plateau of angular fluctuation at the new position is recorded. The right inset shows a closeup of the angular fluctuations around 10 turns. At $\sim 350 \mathrm{~s}$, the magnets are rotated in the opposite direction and the bead returns to its initial equilibrium angle position. (b) Angle fluctuation of a DNA-tethered bead held in conventional magnetic tweezers. Trace for a torsionally relaxed molecule at $\mathrm{N}$ $=0$ turns (dark trace and histogram) and after introducing $\mathrm{N}=40$ turns (light trace and histogram). The solid lines in the right panel are Gaussian fits to the data. Due to the large stiffness of the angular trap in the conventional magnetic tweezers configuration systematic changes in the equilibrium angle are difficult or impossible to detect. (c) Angle fluctuation of a DNA-tethered bead held in magnetic torque tweezers. Trace for a torsionally relaxed molecule at $\mathrm{N}=0$ turns (dark trace and histogram) and after introducing $\mathrm{N}=40$ turns (light trace and histogram). The solid lines in the right panel are Gaussian fits to the data. The restoring torque exerted by the DNA molecule leads to a readily detectable change in the angle distribution.

fluctuations (Eq. (8)): 
The challenge is to reliable detect the changes in equilibrium angle for biologically relevant torques (see, e.g., Croquette and coworkers for an overview ${ }^{16}$ ). As an illustrative example, we consider DNA under a stretching force of $\mathrm{F} \approx 2$ $\mathrm{pN}$, a biologically relevant force and typical situation in magnetic tweezers experiments. If overwound at a stretching force of $2 \mathrm{pN}$, DNA will initially accumulate torsional strain and eventually undergo a buckling transition and form plectonemic supercoils. The critical buckling torque is $\approx 20 \mathrm{pN} \mathrm{nm}$, which is the maximum torque signal expected under these conditions.

In a conventional MT geometry at $\mathrm{F} \approx 2 \mathrm{pN}$, the equilibrium fluctuations have a width of $\sigma_{\theta} \approx 0.9^{\circ}$, corresponding to a trap stiffness of $16600 \mathrm{pN} \mathrm{nm} / \mathrm{rad}$ (Fig. 6(b)). Consequently, the shift in equilibrium angle expected for a torque of $20 \mathrm{pN} \mathrm{nm}$ is $0.0012 \mathrm{rad}$ or $0.07^{\circ}$, which is smaller than our angular resolution. Comparing traces of angular fluctuations at $\mathrm{n}=0$ turns (i.e., torsionally relaxed DNA) and at $\mathrm{n}$ $=40$ turns (which is just past the buckling transition for our $7.9 \mathrm{kbp}$ DNA construct), it is impossible to reliably detect the systematic changes due to the restoring torque exerted by the DNA in the conventional MT configuration (Fig. 6(b)). In general, random scatter in the mean angle by a few tenths of a degree will correspond to torques of $\sim 100 \mathrm{pN} \mathrm{nm}$ for conventional MT, larger than the characteristic torques exerted by DNA. ${ }^{15}$

In contrast, magnets in the MTT configuration provide a much softer angular trap than the conventional MT. Considering again an example traces for a $7.9 \mathrm{kbp}$ DNA construct at $\mathrm{F} \approx 2 \mathrm{pN}$, the width of the equilibrium fluctuations in the MTT is $\sigma_{\theta} \approx 7^{\circ}$, corresponding to a trap stiffness of $\approx 270 \mathrm{pN} \mathrm{nm} / \mathrm{rad}$ (Fig. 6(c)). As a consequence, the restoring torque exerted by the DNA after $n=40$ turns is readily detectable as a systematic shift of angular equilibrium position compared to the $\mathrm{n}=0$ measurement (Fig. 6(c)). In general, torques in the range of $1-10 \mathrm{pN} \mathrm{nm}$ give rise to shifts $\geq 1^{\circ}$ in MTT measurements, enabling the reliably detection of biologically relevant torques using our tracking algorithm. ${ }^{15}$

\section{CONCLUSIONS}

We present a tracking protocol that allows us to determine the $(x, y, z)$-position and rotation angle for beads in MT experiments from analysis of CCD camera images. Evaluation of the tracking accuracy using both simulated images and experimental data indicates an accuracy of $\sim 1-2 \mathrm{~nm}$ in $(\mathrm{x}, \mathrm{y}, \mathrm{z})$ and $0.1^{\circ}$ in angle. The (x, y, z)-resolution is comparable to conventional magnetic tweezers measurements, ${ }^{24,26}$ despite the presence of the fiducial marker bead employed for angular tracking. The angular resolution of $0.1^{\circ}$ is approximately that expected from a simple position resolution argument: two points separated by $\approx 1900 \mathrm{~nm}$ (corresponding to a $0.5 \mu \mathrm{m}$ radius marker beads attached to a $1.4 \mu \mathrm{m}$ radius magnetic bead) that can be localized to within $\approx 2 \mathrm{~nm}$ suggest an angular uncertainty of $\sim 2 \mathrm{~nm} \times 2 \mathrm{~nm} / 1900 \mathrm{~nm} \approx 0.002$ $\operatorname{rad} \approx 0.1^{\circ}$. This simple order-of-magnitude argument is not specific to the details of our tracking algorithm; it generally suggests that it is unlikely for any angular tracking algorithm to yield significantly better resolution than $0.1^{\circ}$, without using much larger fiducial markers than our current implementation for the tracking.

The angular resolution of our algorithm enables the measurements of biologically relevant torques in a MTT scheme, ${ }^{15}$ while maintaining the essentially capabilities of conventional MT, in particular $(\mathrm{x}, \mathrm{y}, \mathrm{z})$ and force resolution. The development of implementations of torque tweezers ${ }^{14-16,18,30}$ is a recent and exciting development that has great potential in a range of biological applications. A particular promising direction is the implementation of massively parallel tracking approaches that monitor tens to hundreds of beads simultaneously. Our tracker is intrinsically multi-bead tracking enabled, the challenges ahead are mostly in optimizing the imaging system and surface functionalization to be able to routinely track large number of beads. In addition, the tracking protocol described here is likely to be useful not only in MTT applications, but for other biophysical measurements as well, for example, in tethered particle motion or for the tracking of only approximately spherical particles.

\section{ACKNOWLEDGMENTS}

We thank Marijn van Loenhout, Iwijn De Vlaminck, John van Noort, Xander Janssen, Leo van IJzendoorn, and Menno Prins for stimulating discussions, Tessa Jager for help with measurements, Jelle van der Does, Dimitri Roos, and Jaap Beekman for help with the instrumentation, and Susanne Hage and Serge Donkers for providing the DNA constructs. This work was supported by Delft University of Technology and by the Netherlands Organisation for Scientific Research (NWO).

${ }^{1}$ T. Strick, J. Allemand, V. Croquette, and D. Bensimon, Prog. Biophys. Mol. Biol. 74(1-2), 115 (2000).

${ }^{2}$ K. C. Neuman and A. Nagy, Nat. Methods 5(6), 491 (2008).

${ }^{3}$ I. D. Vilfan, J. Lipfert, D. A. Koster, S. G. Lemay, and N. H. Dekker, in Handbook of Single-Molecule Biophysics, edited by P. Hinterdorfer and A. van Oijen (Springer, New York, 2009).

${ }^{4}$ M. Manosas, A. Meglio, M. M. Spiering, F. Ding, S. J. Benkovic, F. X. Barre, O. A. Saleh, J. F. Allemand, D. Bensimon, and V. Croquette, Methods Enzymol. 475, 297 (2010)

${ }^{5}$ T. R. Strick, J. F. Allemand, D. Bensimon, A. Bensimon, and V. Croquette, Science 271(5257), 1835 (1996).

${ }^{6}$ T. R. Strick, V. Croquette, and D. Bensimon, Proc. Natl. Acad. Sci. U.S.A. 95(18), 10579 (1998); J. F. Allemand, D. Bensimon, R. Lavery, and V. Croquette, ibid. 95(24), 14152 (1998); A. Crut, P. A. Nair, D. A. Koster, S. Shuman, and N. H. Dekker, ibid. 105(19), 6894 (2008); O. A. Saleh, D. B. McIntosh, P. Pincus, and N. Ribeck, Phys. Rev. Lett. 102(6), 068301 (2009).

${ }^{7}$ J. A. Abels, F. Moreno-Herrero, T. van der Heijden, C. Dekker, and N. H. Dekker, Biophys. J. 88(4), 2737 (2005).

${ }^{8}$ T. R. Strick, V. Croquette, and D. Bensimon, Nature 404(6780), 901 (2000); D. A. Koster, V. Croquette, C. Dekker, S. Shuman, and N. H. Dekker, ibid. 434(7033), 671 (2005).

${ }^{9}$ T. Lionnet, M. M. Spiering, S. J. Benkovic, D. Bensimon, and V. Croquette, Proc. Natl. Acad. Sci. U.S.A. 104(50), 19790 (2007).

${ }^{10}$ B. Maier, D. Bensimon, and V. Croquette, Proc. Natl. Acad. Sci. U.S.A. 97(22), 12002 (2000); A. Revyakin, C. Liu, R. H. Ebright, and T. R. Strick, Science 314(5802), 1139 (2006).

${ }^{11}$ T. R. Strick, M. N. Dessinges, G. Charvin, N. H. Dekker, J. F. Allemand, D. Bensimon, and V. Croquette, Reports Progress Phys. 66(1), 1 (2003).

${ }^{12}$ J. Lipfert, X. Hao, and N. H. Dekker, Biophys. J. 96(12), 5040 (2009).

${ }^{13}$ A. te Velthuis, J. W. J. Kerssemakers, J. Lipfert, and N. H. Dekker, Biophys. J. 99(4), 1292 (2010). 
${ }^{14}$ A. Celedon, I. M. Nodelman, B. Wildt, R. Dewan, P. Searson, D. Wirtz G. D. Bowman, and S. X. Sun, Nano Lett. 9(4), 1720 (2009).

${ }^{15}$ J. Lipfert, J. W. Kerssemakers, T. Jager, and N. H. Dekker, Nat. Methods 7(12), 977 (2010).

${ }^{16}$ F. Mosconi, J. F. Allemand, and V. Croquette, Rev. Sci. Instrum. 82(3), 12 (2011).

${ }^{17}$ Y. Harada, O. Ohara, A. Takatsuki, H. Itoh, N. Shimamoto, and K. Kinosita, Jr., Nature 409(6816), 113 (2001); H. Arata, A. Dupont, J. MineHattab, L. Disseau, A. Renodon-Corniere, M. Takahashi, J. L. Viovy, and G. Cappello, Proc. Natl. Acad. Sci. U.S.A. 106(46), 19239 (2009).

${ }^{18}$ Z. Bryant, M. D. Stone, J. Gore, S. B. Smith, N. R. Cozzarelli, and C. Bustamante, Nature 424(6946), 338 (2003); J. Gore, Z. Bryant, M. D. Stone, M. Nollmann, N. R. Cozzarelli, and C. Bustamante, ibid. 439(7072), 100 (2006).

${ }^{19}$ C. Gosse and V. Croquette, Biophys. J. 82(6), 3314 (2002).

${ }^{20}$ M. Hayashi and Y. Harada, Nucleic Acids Res. 35(19), e125 (2007).

${ }^{21}$ W. P. Wong and K. Halvorsen, Opt. Express 14(25), 12517 (2006); Opt. Lett 34(3), 277 (2009); O. Otto, F. Czerwinski, J. L. Gornall, G. Stober, L. B. Oddershede, R. Seidel, and U. F. Keyser, Opt. Express 18(22), 22722 (2010).
${ }^{22}$ J. Lipfert, D. A. Koster, I. D. Vilfan, S. Hage, and N. H. Dekker, Methods Mol. Biol. 582, 71 (2009).

${ }^{23}$ X. J. Janssen, A. J. Schellekens, K. van Ommering, L. J. van Ijzendoorn, and M. W. Prins, Biosens. Bioelectron. 24(7), 1937 (2009).

${ }^{24}$ T. Strick, Ph.D. thesis, University of Paris VI, 1999.

${ }^{25}$ T. Pilizota, T. Bilyard, F. Bai, M. Futai, H. Hosokawa, and R. M. Berry, Biophys. J. 93(1), 264 (2007).

${ }^{26}$ N. Ribeck and O. A. Saleh, Rev. Sci. Instrum. 79(9), 094301 (2008).

${ }^{27}$ K. Kim and O. A. Saleh, Nucleic Acids Res. 37(20), e136 (2009).

${ }^{28}$ D. Klaue and R. Seidel, Phys. Rev. Lett. 102(2), 028302 (2009).

${ }^{29}$ C. Danilowicz, D. Greenfield, and M. Prentiss, Anal. Chem. 77(10), 3023 (2005); K. Halvorsen and W. P. Wong, Biophys. J. 98(11), L53 (2010).

${ }^{30}$ A. La Porta and M. D. Wang, Phys Rev Lett 92(19), 190801 (2004); S. Forth, C. Deufel, M. Y. Sheinin, B. Daniels, J. P. Sethna, and M. D. Wang, Phys. Rev. Lett. 100(14), 148301 (2008).

${ }^{31}$ M. van Loenhout, J. W. J. Kerssemakers, I. De Vlaminck, and C. Dekker, "Bias-free tracking of spherical particles, enabling nanometer resolution at low magnification" (submitted). 